\title{
ROBUST AND SCALABLE TISSUE-ENGINEERINED ELECTRONIC NERVE INTERFACES (TEENI)
}

Cary A. Kuliasha, , Benjamin S. Spearman ${ }^{1,3}$, Eric W. Atkinson ${ }^{1,4}$, Paritosh Rustogi ${ }^{1,2}$, Abbas S. Furniturewalla ${ }^{1,2}$, Elizabeth A. Nunamaker ${ }^{1,4}$, Kevin J. Otto ${ }^{1,3}$, Christine E. Schmidt ${ }^{1,3}$, and Jack W. Judy ${ }^{1,2,3}$

${ }^{1}$ Nanoscience Institute for Medical and Engineering Technology (NIMET), ${ }^{2}$ Electrical and Computer Engineering,

${ }^{3}$ Biomedical Engineering, and ${ }^{4}$ Neuroscience Departments, ${ }^{4}$ Animal Care Services, University of Florida, Gainesville, Florida, USA

\begin{abstract}
Peripheral nerves are excellent targets for neural interfaces designed to help amputees control prosthetic limbs. However, existing nerve interfaces have designs that significantly limit system performance. To overcome these limitations, we have combined micromachined neural interfaces with tissue-engineered hydrogel-based scaffolds. These tissue-engineered electronic nerve interfaces (TEENI) enable highly scalable nerve interfaces that provide significant interface-design freedom. The use of a hightemperature reactive-accelerated-aging (RAA) soak test sped the identification of a microfabrication processes that can yield robust nerve interfaces. Electrophysiological experiments demonstrate the high-SNR recording performance of chronically implanted TEENI devices.
\end{abstract}

\section{BACKGROUND}

Neurotechnologies that interface portions of the nervous system with implanted electronics have been used to address a wide variety of significant neurological diseases and disabilities. Leading commercialized examples include devices that restore at least a portion of lost sensory function (e.g., cochlear implants to treat deafness, retinal prosthetics to treat forms of blindness, etc.) and devices to recover at least a portion of lost motor function (e.g., deep-brain-stimulation to treat the movement disorders such as Parkinson's disease, peripheral nerve stimulation to reanimate paralyzed limbs, etc.). More recent examples include the use of implantable neurotechnology to stimulate the spinal cord to block pain and to simulate branches of the vagus and other visceral nerves to modulate inflammation and a wide range of autonomic functions. Today, emerging and novel uses of neurotechnology include the treatment of psychiatric conditions (e.g., depression, OCD, PTSD, etc.) and neurologic conditions (e.g., addictions, tinnitus, Tourette's, etc.).

An active field of research is the use of neurotechnology to interface portions of the nervous system with external electronic systems, such as prosthetic limbs for amputees, exoskeletons and other mobility platforms for paralyzed (lower-limb) patients, and computers (e.g., communication, web browsing, etc.) for quadriplegics and other locked-in patients (e.g., ALS, high spinal cord injury, etc.) that are incapable of all (or nearly all) movement. Since these demanding applications benefit from a larger number of channels of independent information, it is not surprising that they have employed the greatest number of electrodes to date (i.e., 100 's to 1000 's) [1].

Although the primary neuroanatomical target for research on neural interfaces intended to support the control of prosthetic limbs has been the motor-control regions of the brain (e.g., primary motor cortex and dorsal premotor cortex), there are drawbacks to this approach. A key drawback is the fact that interfacing with it at high signal bandwidth (e.g., action potentials from individual cells or local fields potentials from small brain regions) requires invasive brain surgery. This may be acceptable, and indeed the only real option, for locked-in patients, but it is unlikely to be acceptable for amputees. Challenging consequences of using the brain as the target for a motor-control interface include the fact that the representation of the motor system is spread over a large area of the cortex (i.e., many $\mathrm{cm}^{2}$ for humans) and that the motorcontrol information is encoded at a higher level of abstraction than the rest of the nervous system. Addressing these challenges can require larger scale systems of greater complexity and likely lower reliability.

\section{INTRODUCTION}

For amputees, the peripheral nerve is a much more attractive target than the brain for several reasons: (1) the surgical procedure has less risk and can be more easily revised if needed, (2) instead of skipping all of the neuroanatomy between the amputation and the somatosensory cortex (i.e., spinal cord, brain stem, various brain regions) the neural sensory and motor information is processed by it, (3) as a result the neural sensory-feedback and motor-control information is encoded at a lower level of abstraction in a nerve (e.g., higher firing rates are proportional to greater muscle contractile force and magnitude of sensory percept), and (4) neural information is constrained to geometrically smaller regions (i.e., the cross sections of one or a few mm-scale nerves).

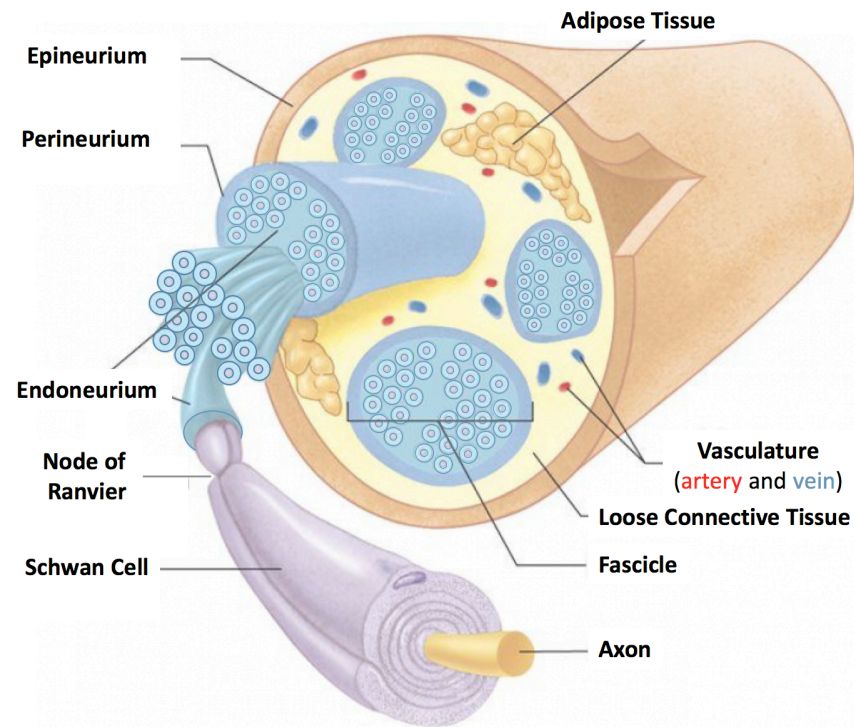

Figure 1. Illustration of the anatomy of a typical peripheral nerve.

Although nerves are not always round, the drawing in Figure 1 highlights key components of the nerve: (a) individual axons that either convey sensory-feedback information up the nerve to the brain from sensory structures or convey motor-control down the nerve to muscles; (b) bundles of axons linked by endoneurium are often grouped into fascicles that are defined by perineurium; (c) 
vasculature to support the functional viability of the nerve; and (d) epineurium around the outside of the nerve to hold it together. Another critical neuroanatomical feature is that all individual sensory and motor axons associated with movement are myelinated (i.e., they are wrapped by a linear sequence of Schwann cells along its length) to support faster signal transmission. The gaps between adjacent insulating Schwann cells along the fibers, known as the Nodes of Ranvier, are where the dynamic transmembrane currents associated with nerve activity are highly localized. Due to the geometric constraints imposed by the nodes on electrical access to the axon, the nodes represent the strongest signal sources for recording neural activity and also the most sensitive targets for stimulating nerve activity. It is for these reasons that the nodes are the primary targets for neural interfaces designed for peripheral nerves. It should be noted that axon diameter varies significantly (e.g., from 1 to $20 \mu \mathrm{m}$ ) depending on their function and that some axons in a nerve are not myelinated at all and thus conduct signals slowly (e.g., pain fibers).

Although the distribution of the nodes is fairly predictable and regular along an individual myelinated axon (i.e., approximately 100 times the diameter of the axons, thus typically $\sim 1 \mathrm{~mm}$ ), there is no correlation of node alignment between neighboring fibers. The result is that the nodes, which are the primary targets of electrophysiological nerve interfaces, are arranged in a roughly repeating 3-D "cloud" inside nerves. Unfortunately, nerve interfaces used to date have 1-D or 2-D designs that do not provide for high channel counts of independent sensory and motor-control information. Since nerve cuffs only have a few large electrodes located outside the circumference of the epineurium, they can only capture compound action potentials (i.e., the combined and overlapping signals from 1000's of axons) and typically have a low signal-to-noise ratio (SNR). Although the polymer-based micromachined longitudinal intrafascicular electrodes (LIFE) or transverse intrafascicular multichannel electrodes (TIME) can record from fewer and even individual axons in a nerve, their 1-D design results in significant undersampling. The silicon-based micromachined Utah slant electrode array (USEA), which consists of rows of probes with increasing length, may appear to interrogate every fiber in a nerve, but upon closer inspection it only samples the 3-D cloud of nodes in a 2-D fashion, thus again undersampling it. Regenerative approaches (e.g., sieve electrode array) also sample nerve activity with a 2-D array of electrodes. What's needed is a nerve-interface approach that comprehensively interrogates nerves with $3-\mathrm{D}$ design and can capture the activity of many individual axons with good SNR.

\section{DESIGN}

To achieve a 3-D nerve interface, one must find a way to create a 3-D arrangement of electrodes inside a nerve. Ideally this interface would be engineered to minimize foreign-body tissue responses that can limit performance and operational lifetime. One way to do this is by making the interface out of extremely compliant materials and geometric designs to avoid a mechanical stiffness mismatch with nerve tissue $(<100 \mathrm{MPa})$, which is hypothesized to trigger an exaggerated foreign-body response to the electrodes that can negatively affect their functional longevity [2]. However, such a mechanically compliant design can be extremely challenging to implant in a dense arrangement.

Our novel multidisciplinary solution is to create mechanically compliant, 3-D scalable, and high-performance nerve interfaces through the combination of microfabricated neural-electronic interfaces and key tissue-engineering methods (e.g., multicomponent nerve scaffolds engineered to facilitate the successful regeneration of cut nerves across gaps larger than $10 \mathrm{~mm}$ ) [3].
Specifically, we have developed a hybrid tissue-engineered electronic nerve interface (TEENI), which consists of multielectrode polyimide-metal-based "threads" embedded into a biodegradable hydrogel composite scaffold that is wrapped in a thin bioresorbable layer of small-intestinal submucosa (SIS) and sutured to the ends of a transected nerve (Figure 2). The multielectrode TEENI threads are supported by a triple-component hydrogel scaffold, which has mechanical properties designed to hold the TEENI threads in place while also reducing foreign-body response. The hydrogel is also designed to degrade gradually during nerve regeneration and to ultimately be replaced by regrown and maturing axons and vasculature [4].

Since the microfabricated electrode array does not need to be forcibly implanted by itself, it does not need to be made from even temporarily stiff materials nor with a straight buckle-resistant design. Also, since the SIS-wrapped scaffold is handled by the surgeons instead of the TEENI threads themselves, the thickness of the polymer-metal threads could be made much thinner than the 10 $\mu \mathrm{m}$ typically required. Although the TEENI shown in Figure 2 has only a 2-D arrangement of electrodes across 4 "threads", it is possible to stack multiple "thread sets" to achieve a 3-D arrangement of electrodes (see upper right inset image). The TEENI approach has enough design flexibility to accommodate nerves of various sizes and cross-sectional geometries and potentially enough scalability to comprehensively engage with the 3 -D distribution of nodes in a nerve.

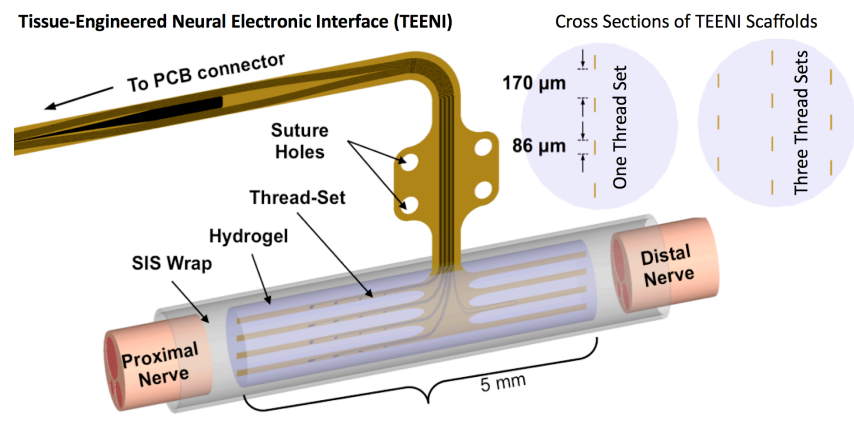

Figure 2: Illustration of a 16-channel TEENI device attached to the ends of a transected nerve. Insets shows 1-mm-diameter crosssectional views of the construct with a single thread set (4) and a multiple thread-set (3-4-3) arrangement.

\section{FABRICATION}

The microfabrication process used to produce the TEENI polymer-metal electrode arrays begins with blank 100-mm diameter silicon wafer. After cleaning in piranha, which forms a thin native oxide layer, the wafers are treated with HMDS and a 5$\mu \mathrm{m}$-thick layer of BPDA-PDA polyimide (PI) precursor (UVarnish $\mathrm{S}, U B E$ Ind.) is formed by spin coating. After the PI is cured $\left(\max\right.$ temp $\left.=350{ }^{\circ} \mathrm{C}\right)$ in a $\mathrm{N}_{2}$ atmosphere, its surface is activated in a reactive-ion-etching (RIE) $\mathrm{O}_{2}$ plasma immediately before a $250-\mathrm{nm}$-thick layer of stoichiometric (1:1 Si:C) a-SiC:H is deposited via PECVD (Unaxis 790, Plasma-Therm, LLC) at 300 ${ }^{\circ} \mathrm{C}$ and $200 \mathrm{~W}$ with $5 \% \mathrm{SiH}_{4} / \mathrm{He}, \mathrm{CH}_{4}$, and $\mathrm{He}$ gasses (Fig 3a). Using a 3- $\mu \mathrm{m}$-thick layer of negative photoresist (nLOF 2035, MicroChemicals $\mathrm{GmbH}$ ) as a lift-off mask, a stack of thin metal layers (Ti: $50 \mathrm{~nm}, \mathrm{Pt}: 100 \mathrm{~nm}, \mathrm{Au}: 100 \mathrm{~nm}, \mathrm{Pt}: 100 \mathrm{~nm}, \mathrm{Ti}: 50 \mathrm{~nm}$ ) are deposited by sputtering (CMS-18 multi-source, Kurt K. Lesker Company) (Fig. 3b). After soaking in NMP at $70{ }^{\circ} \mathrm{C}$ and some sonication, the excess metal is lifted off. A second 250-nm-thick layer of a-SiC:H film is deposited as before (Fig. 3c). Both layers of a-SiC:H are patterned by an $\mathrm{SF}_{6} \mathrm{RIE} / \mathrm{ICP}$ dry etch (Unaxis SLR, 
Plasma-Therm, LLC.) a thin positive photoresist (AZ1512, MicroChemicals $\mathrm{GmbH}$ ) as the etch mask (Fig. 3d). After the residual surface organics are removed by an $\mathrm{O}_{2}$ plasma etch, and an aminopropyl triethoxysilane adhesion agent (VM-652, HD Microsystems) is applied, a second 5- $\mu \mathrm{m}$-thick layer of polyimide precursor is formed by spin coating (Fig. 3e). The Pt electrode sites and connector pads are revealed by an RIE/ICP dry etch in $\mathrm{O}_{2}$ and $\mathrm{SF}_{6}$ plasmas using a $10-\mu \mathrm{m}$-thick layer of positive photoresist (AZ9260, MicroChemicals $G m b H$ ) as an etch mask (Fig. 3f). To form the shape of the TEENI device, both layers of PI are etched in a single $\mathrm{O}_{2}$ plasma etch using a $25-\mu$ m-thick layer of positive photoresist (AZ9260, MicroChemicals $\mathrm{GmbH}$ ) as an etch mask (Fig. 3g). Finally, the TEENI devices are carefully removed from the carrier wafer using tweezers (Fig. 3h).

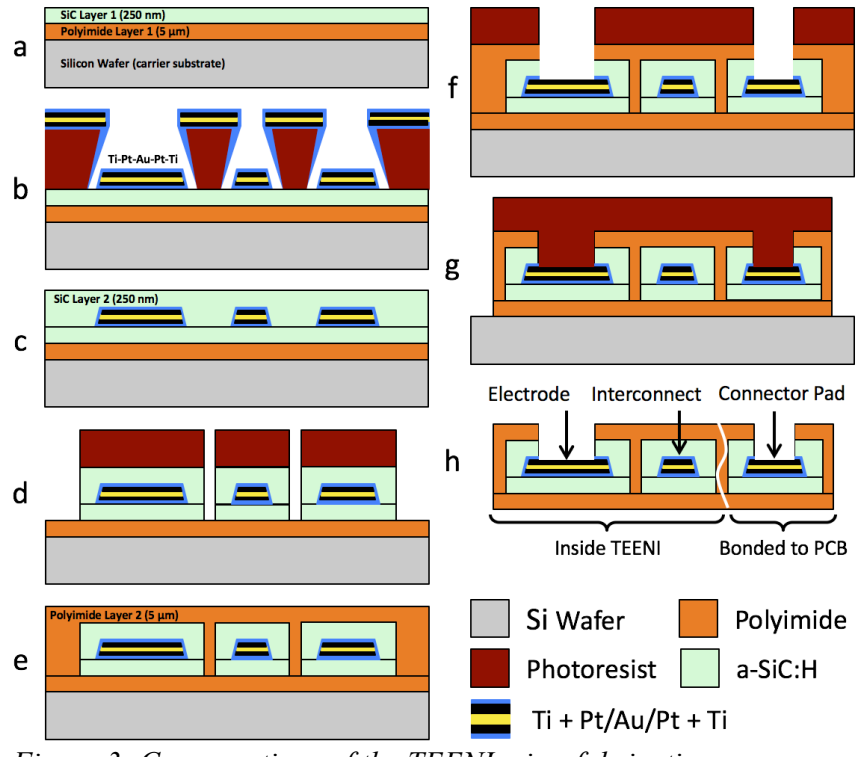

Figure 3: Cross sections of the TEENI microfabrication process.

\section{CHARACERIZATION}

To rapidly assess the robustness of implanted electronic devices, it is common to perform soak tests at elevated temperatures. Given the exponential dependence of thermally driven processes on temperatures (i.e., the Arrhenius relationship), raising the temperature from $\sim 37{ }^{\circ} \mathrm{C}$ (body temperature) to $87{ }^{\circ} \mathrm{C}$ results in an acceleration factor of $\sim 32 \mathrm{X}$ [5]. However, for soak tests to be predictive, the failure modes should be preserved at higher temperatures. For implanted devices, some failures are driven by the aggressive chemical environment (e.g., reactive oxygen species) generated by activated immune cells. However, since the oxygen concentration of saline drops significantly at higher temperatures, it is common for implants to do well with short hot saline-only soak tests and yet fail when implanted for an equivalent time period. A better method is to use a reactiveaccelerated-aging (RAA) soak test that employs hydrogen peroxide as a source of reactive oxygen species to simulate the chemistry of the solution surrounding chronic implants [5].

Since delamination of insulating dielectric layers from the conductive metal layers within neural interfaces has been shown to be a significant failure mode, we used RAA soak tests to rapidly assess different combinations of thin films to promote good adhesion between the polyimide and platinum layers in the TEENI [6]. Specifically, we used 10 to $20 \mathrm{mM}$ of $\mathrm{H}_{2} \mathrm{O}_{2}$ in phosphate buffered saline at $85-87^{\circ} \mathrm{C}$ and tested for 3 days, which is equivalent to a 3.2-month implantation (ASTM F1980). The results shown in Figure 4 illustrate the significant reduction in impedance (at $1 \mathrm{KHz}$ ) observed for different sized electrodes on TEENI made with different combinations of polyimide and various thin adhesion-promoting films. As expected, TEENI made without using an adhesion layer (i.e., for PI-Pt-PI, PI-Pt-Au-Pt-PI) did not maintain electronic integrity (i.e., impedance dropped by 50 to 95\%). Although the addition of titanium as an adhesion layer sometimes resulted in improved outcomes (i.e., impedance dropped by 20 to $30 \%$ ), the combination of silicon carbide and titanium yielded extremely robust results (i.e., impedance changed by $< \pm 5 \%$ ). Figure 5 shows that this robustness exists over the entire frequency band of interest for neural interfaces. Subsequent experiments performed for 7 days confirmed the excellent robustness of the PI-SiC-Ti-Pt-Au-Pt-Ti-SiC-PI material stack inside the TEENI devices. To our knowledge, no other peripheral nerve interface has been soak tested as harshly and survived with excellent functionality.

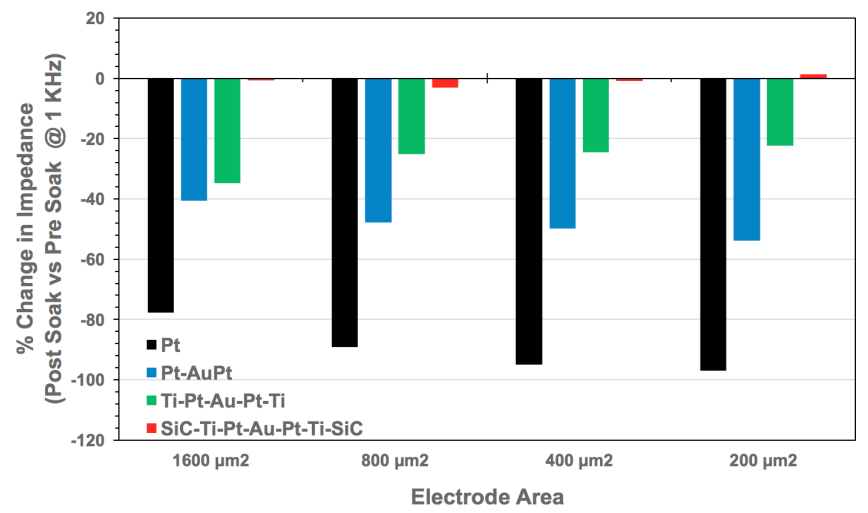

Figure 4. Percent change (reduction) in post-soak impedance (1 KHz) relative to pre-soak values for different sized electrodes.

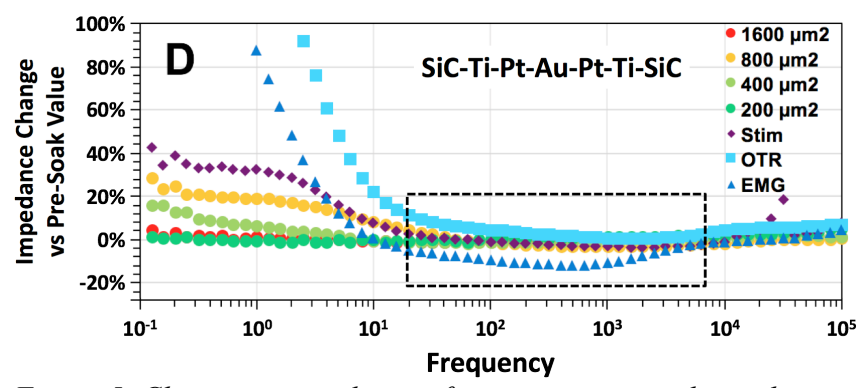

Figure 5. Change in impedance after a reactive-accelerated-aging (RAA) soak test performed at $87^{\circ} \mathrm{C}$ with $20 \mathrm{mM} \mathrm{H} \mathrm{H}_{2} \mathrm{O}_{2}$ for 7 days (estimated to be equivalent to a 7-months implant). When using $\mathrm{SiC}$ and Ti to promote adhesion between the polyimide and the Pt layers, we observed little change in impedance over the frequencies of interest for recording neural signals.

\section{EXPERIMENTAL RESULTS}

After the TEENI devices were microfabricated, they were attached to a small circuit board that soldered to a long bundle of fine wires that are soldered on the other end to an external circuit board with a high-channel-count connector convenient for impedance assessment and electrophysiological measurements. Since the small circuit board attached to the TEENI was to be implanted, it was potted in silicone. The implant was then sterilized and the distal TEENI component was integrated into a biodegradable hydrogel composite scaffold that was wrapped in a bioresorbable small intestinal submucosa (SIS) and sutured to the ends of a transected nerve. In our work we used 250 gm Lewis 
Rats (LEW/Crl) from Charles River Laboratories (Kingston, NY) and formed a 5-mm-long sciatic nerve gaps that were filled by the TEENI device.

Although we were initially surprised to observe strong singleunit recordings (Figure 6) at the distal electrodes after only 4 days, given that the nerve regeneration rate is $\sim 1 \mathrm{~mm}$ /day the results are not that unexpected. Recordings with good SNR were observed for 6 weeks (Figure 7), at which point recording was no longer possible due to head-cap failure (i.e., the structure securing the external connector to the implanted TEENI to the skull).

Explantation of the TEENI device (Figure 8) revealed that they were well integrated into tissue, exhibited extensive

vascularization associated with healthy tissue, and had a significant number and distribution of axons through the scaffold and near the TEENI devices.

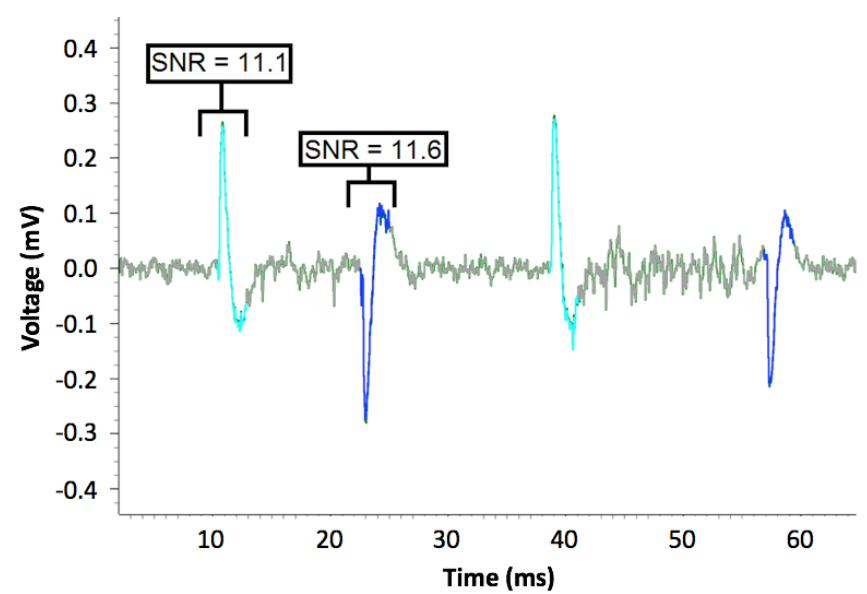

Figure 6. Electrophysiological recording from chronically implanted TEENI device that shows multiple, sortable action potentials from a single electrode.

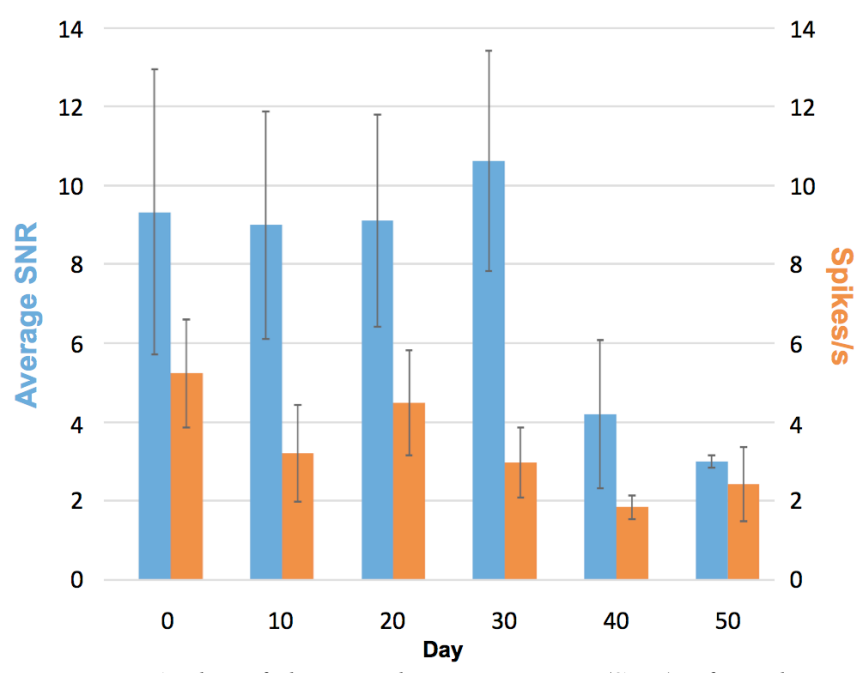

Figure 7: A plot of the signal-to-noise ratio (SNR) of single-unit action potentials and their firing rate versus implant duration.

\section{CONCLUSIONS}

By integrating highly compliant micromachined polymermetal neural interfaces into tissue-engineered scaffolds, it is possible to achieve both a highly scalable and robust nerve interface. Such a nerve-interface design approach is well suited to help amputees control advanced prosthetic limbs with many channels of independent motor-control and sensory-feedback information. Experimental results have shown that single-unit recordings can be obtained from TEENI devices after only 4 days of nerve regeneration and can be maintained for at least several weeks.


Figure 8: Histological analysis of a TEEN device after a 6-week implantation. (left) Optical microscope image of an explanted nerve that regenerated through a TEENI hybrid scaffold with the microfabricated device visible inside the nerve. (top/right) Lightsheet microscope image of a TEENI device (red) and the vasculature (green) inside a regenerated nerve. (center/bottom) Image of regenerated axons within a TEENI device.

\section{ACKNOWLEDGEMENTS}

This work was sponsored by the Defense Advanced Research Projects Agency (DARPA) Biological Technology Office (BTO) Electrical Prescriptions (ElectRx) program under the auspices of Dr. Eric Van Gieson through the DARPA contracts Management Office, Pacific Cooperative Agreement: No. HR0011-15-2-0030. The microfabrication reported here was enabled by the facilities and staff of the University of Florida Research Services Centers.

\section{REFERENCES}

[1] J. Collinger, et al., "High-performance neuroprosthetic control by an individual with tetraplegia", The Lancet, vol. 381, no. 9866 , 2013, pp. 557-564.

[2] R. Biran, et al., "Neuronal cell loss accompanies the brain tissue response to chronically implanted silicon microelectrode arrays”, Exp. Neurol., vol. 195, no. 1, pp. 115-126, Sep. 2005. [2] B.S. Spearman, et al., "Tissue-Engineered Peripheral Nerve Interfaces", Advanced Functional Materials, 2017, vol. 28, no. 12, 2018, p. 1701713.

[4] V. Desai, et al, "Design, fabrication, and characterization of a scalable tissue-engineered-electronic-nerve-interface (TEENI) device", IEEE Neural Engineering Conference, pp. 203-206, 2017. [5] P. Takmakov,..., "Rapid evaluation of the durability of cortical neural implants using accelerated aging with reactive oxygen species", Journal of Neural Engineering, vol. 12, no. 2, 2015. [6] C.A. Kuliasha and J.W. Judy, "in vitro Reactive-AcceleratedAging (RAA) Assessment of Tissue-Engineered Electronic Nerve Interfaces (TEENI)", IEEE EMBC, Orlando, FL, 2016. 